\title{
A MULTI-CRITERIA MODEL FOR SELECTING THE MOST SUITABLE CLASSIFIER FOR SUPPORTING ASSISTIVE TECHNOLOGY ADOPTION IN PEOPLE WITH DEMENTIA
}

\begin{abstract}
The aging population is increasing significantly in developed countries where living standards are high. Aging comes with the inevitable decline in physical and cognitive function that typically requires some form of intervention of self-management to maintain quality of life. In this respect, classifiers were created to evaluate the appropriateness of incorporating assistive technology into everyday living of people with dementia. In this paper, a hybrid fuzzy AHP-TOPSIS method for selecting the most suitable classifier to support and maintain assistive technology adoption in people with dementia is presented. Taking into consideration characteristics related to performance, usability, scalability, flexibility and design of various assistive technologies. This paper discusses the development of decision models, which were based on a number of carefully selected data.
\end{abstract}

Keywords: TOPSIS, FAHP, assistive technology, classifier.

\section{Introduction}

The rapid increase of ageing population is raising concerns in many developed countries, especially in Northern Europe (Figure 1 shows the median ages in Europe). Ageing comes with a number of functional and cognitive declines including dementia. Assistive technology offers the potential to enhance the level of independence of people with dementia, thereby increasing the possibility of supporting home-based care (Zhang et al., 2014). Effective use of such assistive technologies can support people with dementia to perform activities of daily living (ADLs) independently, alleviate the financial burden and potentially improve their quality of life. However, even with such a technology-based solution, a one size fits all solution remains elusive. It is hence relevant to determine the appropriateness for such patients to use assistive technologies. In this regard, several classifiers have been created (e.g. Naive Bayes, Random Forest, and Neural Networks) to detect unsuitability to such technology so that the cost can be reduced and possible negative effects on quality of life of the people with dementia avoided. However, the aptness of these classifiers is influenced by different criteria. Therefore, choosing an appropriate multicriteria decision-making approach has become a critical step in this process. In this respect, although some predictive models have been developed to address this problem (Zhang et al., 2014), scalability, flexibility and design criteria have not been so far taken into account. Additionally, the uncertainty of human judgments when 
prioritizing criteria and sub-criteria has not been considered and is also a contribution of this research.

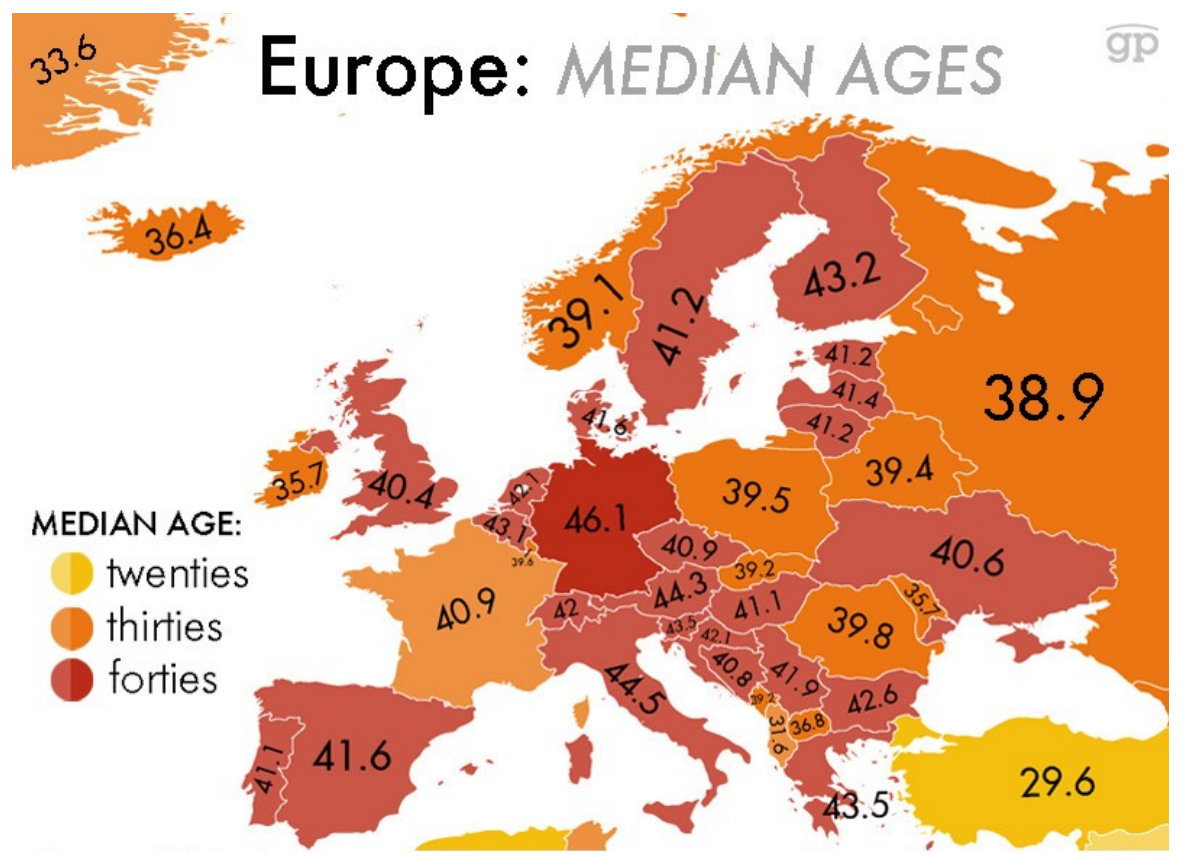

Figure 1: Europe median ages (source: CIA Factbook)

\section{Literature Review}

Home health care services aimed to provide all the necessary help and support while encouraging older people to live independent and dignified lives (Ellenbecker et al., 2008).

There have been many studies carried out into the contribution of technology to elderly care. The technological development towards assistive devices and systems for the elderly became the main focus of researches as the aged population increases alarmingly (Khosravi and Ghapanchi, 2016). In this regard, the classifiers were developed to evaluate the suitability of introducing assistive technology into the everyday living of people with dementia. Choosing an appropriate classifier has then become a critical step in this process. To do this, a number of criteria from different aspects need to be deemed by decision-makers. In this respect, some authors have worked on identifying critical features that may influence technology adoption. Peng et al. (2011) used performance measures for ranking classifiers to be applied in financial risk detection. On the other hand, Zhang et al. (2014) considered prediction performance, robustness, bias with regard to two types of errors and usability for selecting the optimal classifier of assistive technology adoption for people with dementia. In light of these, the studies directly concentrating on this topic are largely limited. In addition, the aforementioned 
studies only consider some criteria when making this decision which may affect the final ranking. Therefore, the research question in this study is: What is the most suitable classifier for assistive technology in supporting self-care of the elderly?

\section{Hypotheses/Objectives}

Classifiers (e.g. $k$-nearest-neighbour $(k N N)$, neural network $(\mathrm{NN})$, support vector machine (SVM), decision tree (DT), adaptive boosting (AB), Naïve Bayes (NB), and CART) were developed for predicting the suitability of incorporating technology in everyday living of people with dementia. In this sense, the classifier can maximize the use of the technology to support independent living. However, several classifiers can be deployed for this purpose, therefore, selecting the best classifier is a crucial aspect to reduce the cost, potential negative effects on quality life and failures during the implementation process. Additionally, it can be regarded as a robust multi-criteria decision making (MCDM) model involving a number of different performance criteria with the ability of discriminating the best classifier alternative.

\section{Research Design/Methodology}

To address this challenge, this paper presents a hybrid fuzzy AHP-TOPSIS method to deal with the selection problem. First, a decision-making team is formed to design the multi-criteria model and identify the performance decision elements (criteria and subcriteria). Following this, fuzzy AHP is used to prioritize criteria under uncertainty (Saaty and Peniwati, 2007). Herein, the team participants (5 experts from Ulster University (Northern Ireland), Halmstad University (Sweden), University of Jaen (Spain) and Lulea Technological University (Sweden)) are asked to make pairwise comparisons (refer to Figure 2) in order to define the importance of each criterion and sub-criterion. After this, TOPSIS is applied to rank the classifiers (Shih et al., 2007) from which the best classifier is identified according to a set of criteria and sub-criteria shown in Section 5.

\begin{tabular}{|c|c|c|c|c|c|c|c|c|}
\hline & & $\mathbf{1}$ & 2 & 3 & 4 & 5 & & \\
\hline Hendling of missingdata & is & $\square$ & $\square$ & $\square$ & $\square$ & $\square$ & Important than & $\begin{array}{l}\text { Handling of continuous } \\
\text { and disaretedtata }\end{array}$ \\
\hline Handling of missingdata & is & $\square$ & $\square$ & $\square$ & $\square$ & $\square$ & Important than & Adaption \\
\hline $\begin{array}{l}\text { Hendlingof continuous } \\
\text { and disaretedata }\end{array}$ & is & $\square$ & $\square$ & $\square$ & $\square$ & $\square$ & Important than & Adaption \\
\hline 1 Muchless & \multicolumn{6}{|c|}{3 Equally } & \multirow{2}{*}{\multicolumn{2}{|c|}{5 Much more }} \\
\hline 2 Less & & & & More & & & & \\
\hline
\end{tabular}

Figure 2. An example of the data-collection tool that used during the decision-making process

\section{Data/Model Analysis}


A decision-making model with 5 criteria, 16 sub-criteria and 7 alternatives (data-mining algorithms) was designed. The model was developed with the aid of the REMIND CONSORTIUM (Ulster University, Halmstad University, and University of Jaen) and studies from the reported literature. Figure 3 shows the proposed decision-making structure. Performance represents the percentage of the correctly classified instances while Usability measures whether the model is easy to understand for healthcare professionals who do not have a computational or technical background. Another criterion of interest is scalability which denotes the financial cost of learning process. On the other hand, the time-window for assistive technology is quite short for people with dementia and the suitability of different technologies will change rapidly over time. Therefore, flexibility and the necessity to evolve (online learning), based on new or emerging parameters relating to the individual's needs is of importance. Finally, design considers the structure of classifiers.

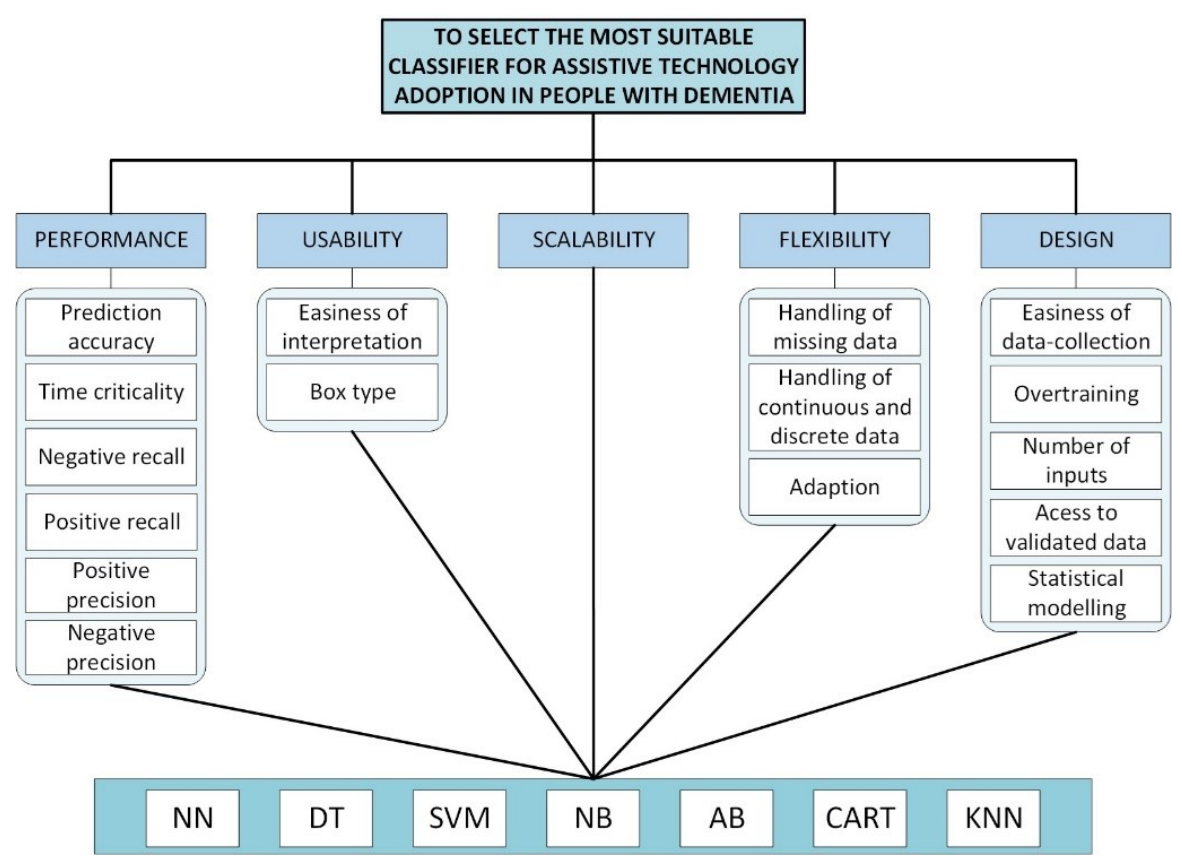

Figure 3: The proposed decision-making structure for selecting the most suitable classifier for assistive technology adoption

From the initial set of results obtained, it is indicated that the most relevant criteria when selecting the most suitable classifier for assistive technology adoption in people with dementia are: SCALABILITY $(\mathrm{GW}=26 \%)$, FLEXIBILITY $(\mathrm{GW}=24 \%)$ and USABILITY $(\mathrm{GW}=23 \%)$. The most important sub-criterion was EASINESS OF INTERPRETATION (18.5\%), which can be underpinned by the need of ensuring that a non-expert (e.g. clinicians) can be able to follow the decision steps that a classifier is making in order to have some confidence that the decision is accurate. On the other hand, after applying FAHP-TOPSIS in a set of seven data-mining algorithms (refer to Figure 3), 
it was concluded that the Naive Bayes) (NB) algorithm is the most suitable classifier with a closeness coefficient of 0.765 (refer to Table 1) Additionally, weaknesses of each classifier were pinpointed in order to support the assistive technology adoption in people with dementia.

\begin{tabular}{|l|c|c|c|c|c|c|c|}
\hline Ranking & 1 & 2 & 3 & 4 & 5 & 6 & 7 \\
\hline Classifier & NB & CART & KNN & DT & NN & AB & SVM \\
\hline $\begin{array}{l}\text { Closeness } \\
\text { coefficien } \\
\text { t }\end{array}$ & 0.765 & 0.710 & 0.561 & 0.492 & 0.487 & 0.409 & 0.273 \\
\hline
\end{tabular}

Table 1. Ranking of classifiers that can be potentially selected for supporting assistive technology adoption in people with dementia

\section{Limitations}

It is essential to mention that the findings could be associated with the case study. This research study was limited to seven data-mining algorithms which may partially explain the outcomes. Future research will consider interrelations and feedback between criteria and the presented methodology will be replicated with other types of assistive technology for people with dementia.

\section{Conclusions}

In general, people with dementia are reluctant to change; therefore, it is important that suitable assistive technologies are selected for them. In fact, assistive technology is proved to have a positive impact on sustaining independence and delaying the longer term care needs. In addition, assistive technology plays important role in preventing functional decline and enabling older people with co-morbidities to cope up with their day to day life routines. Technology has provided variety of products and services. Thus, the main contribution of this paper has been to identify the most relevant criteria when selecting the most suitable classifier for assistive technology adoption in people with dementia.

\section{Acknowledgments}

The Authors which to acknowledge support from the REMIND Project from the European Union's Horizon 2020 research and innovation programme under the Marie Skłodowska-Curie grant agreement No 734355.

\section{Key References}

Ellenbecker, C.H., Porell, F,W, Samia, L., Byleckie, J.J., Milburn, M. (2008). Predictors of Home Healthcare Nurse Retention. Journal of Nursing scholarship, 40(2), 151-160. 
ISAHP Article: A Style Guide for Paper Proposals To Be Submitted to the International Symposium on the Analytic Hierarchy Process 2018, Hong Kong, HK.

Khosravi, P., \& Ghapanchi, A.H. (2016). Investigating the effectiveness of technologies ap-plied to assist seniors. International Journal of medical informatics, (85), 17-26.

Peng, Y., Wang, G., Kou, G., \& Shi, Y. (2011). An empirical study of classification algorithm evaluation for financial risk prediction. Applied Soft Computing, 11(2), 29062915.

Saaty, T.L., \& Peniwati, K. (2007). Group decision-making: Drawing out and reconciling differences. Pittsburgh, PA: RWS Publications.

Shih, H., Shyur, H., Lee, E. (2007). An extension of TOPSIS for group decision making. Mathematical and Computer Modelling, 45, 801-813.

Zhang S., McClean S.I., Nugent C.D., Donnelly M.P., Galway L., Scotney B.W., Cleland I. (2014). A predictive model for assistive technology adoption for people with dementia. IEEE J Biomed Health Inform, 18(1), 375-83. 